\title{
Impact of integrated use of diagnostic ultrasound examinations in respiratory intensive care units
}

\author{
Taher A. Al Najjar ${ }^{a}$, Ashraf M. Madkour ${ }^{\mathrm{a}}$, Nehad M. Osman ${ }^{\mathrm{a}}$, \\ Ashraf A. Gomaa ${ }^{\mathrm{a}}$, Ahmed M. Osman ${ }^{\mathrm{b}}$, Mohammed F. El Bagalaty ${ }^{\mathrm{a}}$, \\ Khaled A. Abd EL Kader ${ }^{\mathrm{C}}$
}

\begin{abstract}
Background Implementing point-of-care multiorgan ultrasound (POCUS) to the initial assessment of ICU patients allows intensivists to immediately integrate ultrasound findings with the patient history, physical, and laboratory results, yielding a powerful clinical synergy, improving diagnostic accuracy, and ameliorating further management plans. The aim of this work was to assess the diagnostic performance and therapeutic effect of POCUS in patients admitted to respiratory ICU (RICU).
\end{abstract}

Patients and methods A prospective study was carried out on patients admitted to the RICU. POCUS examination was performed to the patients within $12 \mathrm{~h}$ of admission that included echocardiography, lung ultrasound, abdominal ultrasound including inferior vena cava assessment and lower limb venous duplex.

Results A total of 102 patients were included. The total number of sonographic findings was 320, of which 94 (29.3\%) were new findings. This resulted in confirmation of the admitting diagnosis, modification of the admitting diagnosis, prompted further testing, change in medical therapy prescribed, and prompted invasive procedures in $35,51,11$, 41 , and $14 \%$ of patients, respectively. However, it was ineffective in confirming or modifying diagnosis, provided

\section{Introduction}

The inaccuracy of physical examination at admission to the ICU has been extensively reported, and lifethreatening conditions could be missed at the primary assessment, especially in patients presented with acute respiratory symptoms [1-3]. Thus, intensivists need to make rapid, accurate, and appropriate decisions in situations where there is a high degree of stress and uncertainty and when patients possess little physiologic reserve [4].

Implementing point-of-care multiorgan ultrasound (POCUS) allows the intensivists to personally perform and interpret the ultrasound (US) examination results at the bedside and immediately integrate ultrasound findings with the patient history, physical, and laboratory results, hence yielding a powerful clinical synergy, improving diagnostic accuracy and ameliorating further management plans [5-8].

Results of several studies have shown that application of POCUS in patients admitted with acute respiratory symptoms to emergency department or wrong diagnosis, and missed a diagnosis in 29.4, 2, and $11.7 \%$ of patients, respectively.

Conclusion Integrating POCUS in the initial assessment of critically ill RICU patients together with standard diagnostic tests lead to diagnostic and therapeutic changes in most of patients which affected the management of these patients. Thus, it seems reasonable to consider the routine use of POCUS as a new respiratory examination option in the armamentarium of the intensivists.

Egypt J Bronchol 2018 12:448-460

(C) 2018 Egyptian Journal of Bronchology

Egyptian Journal of Bronchology 2018 12:448-460

Keywords: bedside ultrasound; echocardiography; point of care; respiratory intensive care

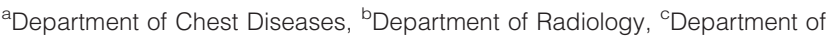
Cardiology, Faculty of Medicine, Ain Shams University, Cairo, Egypt

Correspondence to Mohammed Fathi El Bagalaty, MSc Chest Diseases, Assistant Lecturer of Pulmonary Medicine and Critical Care Faculty of Medicine, Ain Shams University, 12 Soliman al Tamawy st, Sheraton Buildings, Cairo, Egypt. Tel: +20 1007088 660;

e-mail: Mohammedfathi87@gmail.com

Received 29 July 2018 Accepted 12 August 2018

ICU was superior to standard diagnostic tests alone for establishing an accurate diagnosis, leading to changes in medical therapy and prompting further invasive procedures [3,5,8]. Therefore, now it seems crucial to use POCUS as part of the standard diagnostic tests in critically ill patients.

Lung US has been recently introduced in our respiratory ICU (RICU). Its effect on diagnosis and management has been established in several studies $[9,10]$. Combing lung US examination with other organ US examinations, such as heart, inferior vena cava (IVC), abdomen, and deep venous system, using POCUS concept into standard diagnostic assessment of critical ICU patients, remains scarcely applied on the international level and has never been applied in our RICU [11,12]. The actual levels of POCUS implementation and contribution

This is an open access journal, and articles are distributed under the terms of the Creative Commons Attribution-NonCommercial-ShareAlike 4.0 License, which allows others to remix, tweak, and build upon the work non-commercially, as long as appropriate credit is given and the new creations are licensed under the identical terms. 
to ICU patient management need further studies $[11,13]$.

The aim of our study was to assess the diagnostic performance and therapeutic effect of POCUS in patients admitted to RICU.

\section{Patients and methods}

\section{Setting and study population}

This prospective study was carried out on patients admitted to the RICU of Ain Shams University hospitals, Egypt, in the period from October 2016 to July 2017. The study design has been approved by the research ethics committee of our institution, and a written informed consent was obtained from the patients or their relatives.

\section{Inclusion and exclusion criteria}

All adult patient admitted to the RICU were included when the sonographic examinations could be performed within $12 \mathrm{~h}$ after the primary assessment. Exclusion criteria were age younger than 18 years, discharge form RICU within $24 \mathrm{~h}$, and the presence of patient-related conditions that strongly hamper ultrasound examination such as morbid obesity, the presence of subcutaneous emphysema and severe edema, and the presence of environmentrelated limitations that hamper proper examination such as patient isolation for fear of infection.

\section{Primary assessment}

On admission, the diagnosis was made by the attending resident and senior registrar in charge, who had at least 3 years of experience in RICU, depending on history taken in the emergency department or referring ward, clinical examination, laboratory findings, and imaging findings without beside ultrasonographic evaluation. The severity of the patient's condition on admission was graded using the new Simplified Acute Physiology Score (SAPS II) [14]

\section{Sonographic examination}

Within $12 \mathrm{~h}$ of RICU admission, POCUS of the heart, lungs, deep veins, and abdomen was performed by a single physician (M.F.) who received a 2 -month comprehensive training in bedside ultrasonography and echocardiography under supervision of an expert radiologist and an expert cardiologist. This was followed by a 2-month period of directly supervised practice after which he started to work independently.
The operator was aware of the patient's clinical picture but was blinded from the provisional diagnosis and any initial radiological assessment.

Ultrasound machine used was MINDRAY M7 Ultrasound machine (Mindray Bio-Medical Electronics Co., Shenzhen, China), equipped with a linear probe $(5-10 \mathrm{MHz})$, a sector probe $(2-4 \mathrm{MHz})$, and a convex probe $(2-5 \mathrm{MHz})$. No particular order was recommended for the examination.

\section{The examinations included}

Lung ultrasound

'Six' ultrasound areas were examined on each side: the anterior, lateral, and posterolateral views in the upper and lower thoracic regions [2]. Interpretation was done following the principles described by Lichtenstein [15]. The low-frequency curvilinear probe was used to examine the lung parenchyma at a depth of $10 \mathrm{~cm}$ and the high-frequency linear probe for examination of pleural sliding on 2D and M-mode.

\section{Focused echocardiography}

A goal-directed transthoracic echocardiography was done using the basic views, including parasternal short axis and long axis views, apical views (fourchamber, five-chamber, and two-chamber), and subcostal views [2].

\section{Pelvi-abdominal ultrasound}

Pelvi-abdominal ultrasound was done according to 'the focused assessment with sonography for trauma' examination [16].

\section{Assessment of the inferior vena cava}

The subcostal view was used to measure maximum diameter, estimate the percent of respiratory collapsibility (Caval index) [17], and visualize the intraluminal thrombosis. The curvilinear $2-5-\mathrm{MHz}$ probe was used. Measurements were made at a distance not less than $2 \mathrm{~cm}$ caudal from the junction of the right atrium [18].

\section{Venous system}

Mild compression maneuver was used to assess the lower limb (right and left femoral and popliteal veins) and neck vessels (right and left jugular veins). Doppler study was used when needed.

The following specific diagnostic points (Table 1 ) were prospectively defined as previously described by others $[2,3]$. 
Table 1 Prospective definition of specific diagnostic points

\begin{tabular}{l} 
Clinical diagnosis \\
\hline Pneumothorax \\
Pneumonia \\
Cardiogenic pulmonary \\
(increased PV hydrosta \\
pressure) \\
ARDS/ALI \\
Chronic Interstitial lung \\
disease
\end{tabular}
disease

Pleural effusion

Asthma/COPD/normal lung aeration

Clinical diagnosis

Valvular disease

Systolic heart failure

LV, LA dilatation

Pulmonary hypertension

Cor pulmonale

Pericardial effusion

Valve vegetation

Clinical diagnosis

Peritoneal ascites

Cholecystitis

Hydronephrosis

Thoracic examination

Absence of 'lung sliding', absence of B-lines, and detection of the 'lung point'

One of the 4 profiles: $C$ profile,

B' Profile, A/B profile, and A-nov-PLAPS profile

More than 3 B-lines/examined area; extended from the lung bases to the medium and superior fields, bilaterally, symmetrically, without pleural line abnormalities

Nonhomogeneous B-line distribution (more than 3 B-lines/ examined area); presence of spared areas and pleural line abnormalities; and subpleural consolidations

Heterogenous B-line distribution usually more at bases (more than 3 B-lines/examined area); presence of pleural line irregularity and may show subpleural alteration

Echogenic or echo-free space between the visceral and parietal pleura, which may be: Anechoic effusion, Complex nonseptated, Complex septated pattern with fibrin strands and septations within or the homogenously echogenic pattern

Nude profile (Bilateral A lines with lung sliding and no DVT

Cardiac examination

Moderate/severe valvular insufficiency/stenosis EF less than $45 \%$

LA more than $5 \mathrm{~cm}$, LV more than $6 \mathrm{~cm}$

Peak systolic pressure greater than $30 \mathrm{mmHg}$

Altered structure and/or impaired function of the right ventricle that results from pulmonary hypertension associated with diseases of the lung, upper airway, or chest wall

Moderate/severe pericardial effusion more than $2 \mathrm{~cm}$

Mobile hypoechoic soft tissue lesion resting on the valve

Abnormal abdomen examination

An echoic or echogenic with floating particles

Gallbladder distension, pericholecystic fluid, gallbladder wall more than $3.5 \mathrm{~mm}$, and ultrasound Murphy's sign

Dilated pelvis and collecting system, hypoechoic area in the kidney hilum

(Continued) and no PLAPS)
Table 1 (Continued)

\begin{tabular}{lc}
\hline Clinical diagnosis & Thoracic examination \\
\hline $\begin{array}{l}\text { Parenchymal abnormalities } \\
\text { (spleen, liver, kidney, and } \\
\text { bladder) }\end{array}$ & $\begin{array}{c}\text { Parenchymal abnormalities such } \\
\text { as liver cirrhosis, focal lesions, } \\
\text { and nephropathy, and bladder } \\
\text { assessment for retention } \\
\text { Abnormal venous system } \\
\text { examination }\end{array}$ \\
DVT positive vein & $\begin{array}{c}\text { Distended non-compressible } \\
\text { vein, filled with echogenic } \\
\text { material (thrombus) }\end{array}$ \\
& $\begin{array}{c}\text { Inferior vena cava (IVC) } \\
\text { assessment }\end{array}$ \\
CVP $>10$ mmHg & diameter $>2$ cm and absent or \\
reduced $(<50 \%)$ collapsibility \\
diameter $<2 \mathrm{~cm}$ and total or
\end{tabular}

ALI, acute lung injury; ARDS, adult respiratory distress syndrome; COPD, chronic obstructive pulmonary disease; CVP, central venous pressure hypertrophy; DVT, deep vein thrombosis; EF, ejection fraction; LA, left atrium; LV, left ventricle; RA, right atrium; $\mathrm{RV}$, right ventricle.

We used the criteria previously described by Manno et al. [2] to define ultrasound-induced modification, confirmation, wrong evaluation, and lack of confirmation of admitting diagnosis (Table 2).

Ultrasound findings not previously known to the attending resident and the senior registrar in charge, which was unrevealed by ultrasonographic examination, will be defined as a 'new' finding. Changing the admitting diagnosis or medical therapy or to perform invasive procedures was decided by the senior physician and the RICU consultant.

\section{Statistical analysis}

Data were collected, revised, coded, and entered to the Statistical Package for Social Science (IBM SPSS) version 20.0. (SPSS Inc., Chicago, Illinois, USA). Descriptive statistics were performed, including demographic characteristics, medical history, and symptoms, at admission. The effect of point of care ultrasonography on the diagnosis and treatment was calculated. Percentages were computed for the categorical variables. Data analysis was conducted using SPSS software, version 20.0 (SPSS Inc., Chicago, Illinois, USA).

\section{Results}

\section{Baseline patient characteristics}

A total of 102 patients were enrolled in our study. Patient characteristics, presenting symptoms, and 
Table 2 Criteria to define ultrasound-induced modification, confirmation, wrong evaluation, and lack of confirmation of admitting diagnosis

\begin{tabular}{lc}
\hline $\begin{array}{l}\text { Ultrasound-induced } \\
\text { modification of admitting } \\
\text { diagnosis }\end{array}$ & $\begin{array}{l}\text { (a) Ultrasound evidence of an } \\
\text { etiological diagnosis (unknown) } \\
\text { upon a generic organ failure. (b) } \\
\text { Ultrasound allows a different } \\
\text { etiological diagnosis in } \\
\text { comparison with the etiological } \\
\text { admitting diagnosis }\end{array}$ \\
$\begin{array}{l}\text { Ultrasound-induced } \\
\text { confirmation of admitting } \\
\text { diagnosis }\end{array}$ & $\begin{array}{c}\text { admitting diagnosis } \\
\text { Ultrasound-induced wrong } \\
\text { evaluation of diagnosis }\end{array}$ \\
$\begin{array}{l}\text { (a) Ultrasound-based etiological } \\
\text { diagnosis was not confirmed by } \\
\text { gold standard. (b) Ultrasound } \\
\text { missed etiological diagnosis } \\
\text { evidenced by gold standard }\end{array}$ \\
$\begin{array}{l}\text { Ultrasound was not effective in } \\
\text { diagnosis by ultrasound }\end{array}$ & confirming or modifying etiological \\
& diagnosis
\end{tabular}

medical history are presented in Table 3. Overall, 18 $(17.6 \%)$ patients required invasive mechanical ventilation and nine patients needed noninvasive positive pressure ventilation.

The admitting and final diagnosis among studied patients are reported in Table 4; Acute exacerbation of chronic obstructive pulmonary disease was the most common diagnosis among our patients.

Different sonographic findings are shown in Table 5. The total number of sonographic findings was 320, of which 94 (29.3\%) were new ultrasound findings (Figs 1-3). The number of new finding per patient ranged from 0 to 4 with a median of one finding per patient.

Regarding the IVC assessment, 35 (34.3\%) patients had plethoric IVC, another two (2\%) patients showed signs of acute overload (sludge), 20 (19.6\%) patients had IVC with signs of intravascular volume depletion, and IVC could not be assessed in seven (6.8\%) patients.

\section{Diagnostic effect}

The effect of ultrasound examination among studied patients is detailed in Table 6. Ultrasound examination modified the admitting diagnosis in 52/102 cases (50.9\%) (Figs 1 and 5). In 14 (13.7\%) cases, more than one modality was used. Ultrasound also confirmed the diagnosis (Figs 2 and 3) in 36/102 (35.2\%) cases, was ineffective in confirming or modifying in 30 (29.4\%) of 102 cases, had a wrong diagnosis (Fig. 4) in $2(2 \%)$ of 102 , and missed a diagnosis in 12 (11.7\%) of 102 cases.
Table 3 Patient demographics

\begin{tabular}{|c|c|}
\hline & $N=102[n(\%)]$ \\
\hline \multicolumn{2}{|l|}{ Age (years) } \\
\hline Mean \pm SD & $53.59 \pm 16.61$ \\
\hline Range & 19-90 \\
\hline Median & 57 \\
\hline \multicolumn{2}{|l|}{ Sex } \\
\hline Male & $69(67.6)$ \\
\hline Female & $33(32.4)$ \\
\hline \multicolumn{2}{|l|}{ Mechanical ventilation } \\
\hline No MV & $75(73.5)$ \\
\hline Invasive MV & $18(17.6)$ \\
\hline Noninvasive MV & $9(8.8)$ \\
\hline \multicolumn{2}{|l|}{ Relevant history } \\
\hline Intravenous drug addict & 5 \\
\hline Pregnancy & 3 \\
\hline \multicolumn{2}{|l|}{ Presenting symptoms } \\
\hline Dyspnea & 91 \\
\hline Orthopnea & 8 \\
\hline Wheeze & 9 \\
\hline Cough and expectoration & 43 \\
\hline Dry cough & 13 \\
\hline Hemoptysis & 18 \\
\hline Fever/toxic symptoms & 20 \\
\hline Chest pain & 8 \\
\hline Disturbed conscious level & 7 \\
\hline Generalized edema & 6 \\
\hline Cyanosis & 3 \\
\hline
\end{tabular}

$\mathrm{MV}$, mechanical ventilation.

The ultrasonographic findings prompted further testing (Fig. 5) in 11/102 (10.8\%) patients, changes in medical therapy in 42/102 (41.2\%) patients, and led to invasive procedures (Fig. 6) in 14/102 (13.7\%) patients.

\section{Sonographic examination}

The time required to complete the examination ranged from 15 to $45 \mathrm{~min}$, being least in the last 20 patients, with a mean of $38.4 \mathrm{~min}$.

\section{Outcome}

Regarding patient outcome, 79/102 (77.5\%) of the patients improved and were discharged, 18/102 (17.6\%) died, and 5/102 (4.9\%) were transferred to other hospitals.

\section{Discussion}

Adding POCUS of the heart, lungs, abdomen, IVC, and deep veins to the standard initial diagnostic tests within $12 \mathrm{~h}$ of RICU admission resulted in confirmation of the admitting diagnosis, modification of the admitting diagnosis, prompted further testing, change in medical therapy prescribed, and prompted invasive procedures in 35, 
Table 4 Admitting and final diagnosis among studied patients

\begin{tabular}{|c|c|c|c|}
\hline \multirow{2}{*}{$\begin{array}{l}\text { Admitting diagnosis } \\
\text { AECOPD }\end{array}$} & \multicolumn{3}{|c|}{ Final diagnosis } \\
\hline & 28 & AECOPD & 23 \\
\hline & & AECOPD+lung cancer & 2 \\
\hline & & AECOPD+bronchiectasis & 1 \\
\hline & & Cardiogenic pulmonary edema & 2 \\
\hline Acute severe asthma & 3 & Acute severe asthma & 3 \\
\hline Bronchiectasis acute exacerbation & 5 & Bronchiectasis acute exacerbation & 5 \\
\hline $\begin{array}{l}\text { Diffuse parenchymal lung disease with respiratory } \\
\text { failure }\end{array}$ & 8 & Diffuse parenchymal lung disease with respiratory failure & 8 \\
\hline Acute cardiogenic pulmonary edema & 2 & Acute cardiogenic pulmonary edema & 2 \\
\hline ARDS & 2 & ARDS & 2 \\
\hline \multirow[t]{6}{*}{ Lower respiratory tract infection } & 16 & Pneumonia & 10 \\
\hline & & $\begin{array}{l}\text { Postobstructive pneumonia secondary to endobronchial carcinoid } \\
\text { tumor }\end{array}$ & 1 \\
\hline & & Cardiogenic Pulmonary edema & 2 \\
\hline & & Lung cancer & 1 \\
\hline & & Pulmonary embolism & 1 \\
\hline & & IEC & 1 \\
\hline \multirow[t]{4}{*}{ Suspected pulmonary embolism } & 9 & Pulmonary embolism & 6 \\
\hline & & Pulmonary TB & 1 \\
\hline & & Lung cancer & 1 \\
\hline & & Cardiogenic pulmonary edema & 1 \\
\hline Primary pulmonary hypertension & 1 & Primary pulmonary hypertension & 1 \\
\hline Empyema & 3 & Empyema & 3 \\
\hline \multirow[t]{2}{*}{ Abscess } & 3 & Abscess & 2 \\
\hline & & IEC+abscess & 1 \\
\hline \multirow[t]{2}{*}{ Lung cancer } & 8 & Lung cancer & 7 \\
\hline & & Lung cancer+tamponading malignant pericardial effusion & 1 \\
\hline Pleural mesothelioma & 2 & Mesothelioma & 2 \\
\hline \multirow[t]{2}{*}{ OHVS/OSA } & 4 & OHVS/OSA & 3 \\
\hline & & Cardiogenic pulmonary edema & 1 \\
\hline \multirow[t]{2}{*}{ Shock } & 2 & Cardiogenic shock & 1 \\
\hline & & Hypovolemic shock & 1 \\
\hline \multirow[t]{3}{*}{ Hemoptysis } & 4 & Tight MS & 1 \\
\hline & & Pulmonary TB & 1 \\
\hline & & Pneumonia & 2 \\
\hline \multirow[t]{2}{*}{ Undiagnosed pleural effusion with respiratory failure } & 2 & Lung cancer & 1 \\
\hline & & Tamponading malignant pericardial effusion & 1 \\
\hline Total & 102 & Total & 102 \\
\hline
\end{tabular}

AECOPD, acute exacerbation of COPD; ARDS, acute respiratory distress syndrome; IEC, infective endocarditis; MS, mitral stenosis; OHVS, obesity hypoventilation syndrome; OSA, obstructive sleep apnea; PPHTN, primary pulmonary hypertension; PVC, pulmonary venous congestion; TB, tuberculosis.

$51,11,41$, and $14 \%$ of patients, respectively. However, it was ineffective in confirming or modifying diagnosis, provided wrong diagnosis, and missed a diagnosis in $29.4,2$, and $11.7 \%$ of patients, respectively.

Several previous studies evaluated POCUS in patients presented with respiratory symptoms to the emergency room (ER) or general ICUs, but up to our knowledge, none of them assessed it specifically in RICU $[2,3,5,8,12,13]$. RICU patients are a heterogeneous group presenting with either primary respiratory disease or secondary respiratory disease to other illness. They are characterized by respiratory failure, need for mechanical ventilation, severe illness, multiple system dysfunction, and multiple coexisting comorbidities [19].

In a prospective multicentric study in 142 ICUs in France, Belgium, and Switzerland by Zieleskiewicz et al. [13] to describe the diagnostic and therapeutic effects of POCUS performed during a 24-h period, the use of POCUS changed the diagnosis in $21 \%$ of cases, led to confirmation of a suspected diagnosis in $63 \%$ of cases, and was associated with interventions including treatment, imagery ordering, and patient triage in $69 \%$ of cases [13]. 


\begin{tabular}{|c|c|c|}
\hline & All findings $(N=320)[n(\%)]$ & $\operatorname{New}(N=94)[n(\%)]$ \\
\hline Lung ultrasound & $130(40.6)$ & $12(12.8)$ \\
\hline \multicolumn{3}{|l|}{ Asthma/COPD/normal lung aeration } \\
\hline Nude profile (A-profile with no DVT and no PLAPS) & $26(8.1)$ & \\
\hline \multicolumn{3}{|l|}{ Pulmonary embolism } \\
\hline A-DVT-profile & $3(0.9)$ & \\
\hline \multicolumn{3}{|l|}{ Alveolar interstitial syndrome (B profile) } \\
\hline Cardiogenic pulmonary edema & $13(4.1)$ & $6(6.4)$ \\
\hline ARDS/ALI & $3(0.9)$ & \\
\hline Interstitial syndrome & $7(2.2)$ & $2(2.1)$ \\
\hline Bronchiectasis & $4(1.3)$ & $1(1.1)$ \\
\hline \multicolumn{3}{|l|}{ Pneumonia } \\
\hline C profile & $16(5.0)$ & \\
\hline B'-profile & $10(3.1)$ & \\
\hline $\mathrm{A} / \mathrm{B}$ & $1(0.3)$ & \\
\hline A-noV-PLAPS-profile & $3(0.9)$ & \\
\hline \multicolumn{3}{|l|}{ Pleural abnormalities } \\
\hline Simple unilateral pleural effusion & $16(5.0)$ & $1(1.1)$ \\
\hline Simple bilateral pleural effusion & $7(2.2)$ & \\
\hline Complex pleural effusion & $7(2.2)$ & \\
\hline Complex septated effusion & $2(0.6)$ & $2(2.1)$ \\
\hline Pleural thickening & $1(0.3)$ & \\
\hline Pneumothorax (A' profile) & $1(0.3)$ & \\
\hline \multicolumn{3}{|l|}{ Focal lesions } \\
\hline Infarction/wedges & $5(1.6)$ & \\
\hline Lung mass & $4(1.3)$ & \\
\hline Abscess & $1(0.3)$ & \\
\hline Echocardiography & $150(46.9)$ & $62(66.0)$ \\
\hline Valvular heart disease & $15(4.7)$ & $7(7.4)$ \\
\hline PHTN & $52(16.3)$ & $14(14.9)$ \\
\hline Dilated RV/RA with preserved RV function & $21(6.6)$ & $5(5.3)$ \\
\hline Impaired RV systolic function & $15(4.7)$ & $7(7.4)$ \\
\hline EF $45 \%$ or less & $19(5.9)$ & $13(13.8)$ \\
\hline LV/LA dilatation & $14(4.4)$ & $6(6.4)$ \\
\hline Pericardial effusion & $11(3.4)$ & $7(7.4)$ \\
\hline Valve vegetations & $2(0.6)$ & $2(2.1)$ \\
\hline Atrial Invasion & $1(0.3)$ & $1(1.1)$ \\
\hline Pelviabdominal ultrasound & $31(9.7)$ & $14(14.9)$ \\
\hline Organomegally & $6(1.9)$ & $2(2.1)$ \\
\hline Ascites & $9(2.8)$ & $4(4.3)$ \\
\hline Bladder mass & $1(0.3)$ & $1(1.1)$ \\
\hline Cholecystitis & $1(0.3)$ & $1(1.1)$ \\
\hline Nephropathy & $2(0.6)$ & \\
\hline Hydronephrosis & $1(0.3)$ & $1(1.1)$ \\
\hline Cystic kidney & $4(1.3)$ & $1(1.1)$ \\
\hline Hepatic focal lesion & $5(1.6)$ & $3(3.2)$ \\
\hline Chronic liver disease & $1(0.3)$ & \\
\hline Cystic liver & $1(0.3)$ & $1(1.1)$ \\
\hline Duplex & $9(2.8)$ & $6(6.4)$ \\
\hline Lower limb DVT & $4(1.3)$ & $4(4.3)$ \\
\hline IJV thrombosis & $5(1.6)$ & $2(2.1)$ \\
\hline
\end{tabular}

ALI, acute lung injury; ARDS, adult respiratory distress syndrome; DVT, deep venous thrombosis; EF, ejection fraction; IJV, internal jugular vein; LA, left atrium; LV, left ventricle; M/AVD, mitral/aortic valve disease; PHTN, pulmonary hypertension; RA, right atrium; RV, right ventricle; TR, tricuspid regurgitation.

Manno et al. [2] also investigated the use of POCUS in ICU patients, and it was found that ultrasound examination confirmed the admitting diagnosis in
$58.4 \%$ of cases, modified it in $25.6 \%$ of cases, was ineffective in confirming or modifying it in $13.6 \%$ of cases, and missed it in $2.4 \%$ of cases. The 


\section{Figure 1}

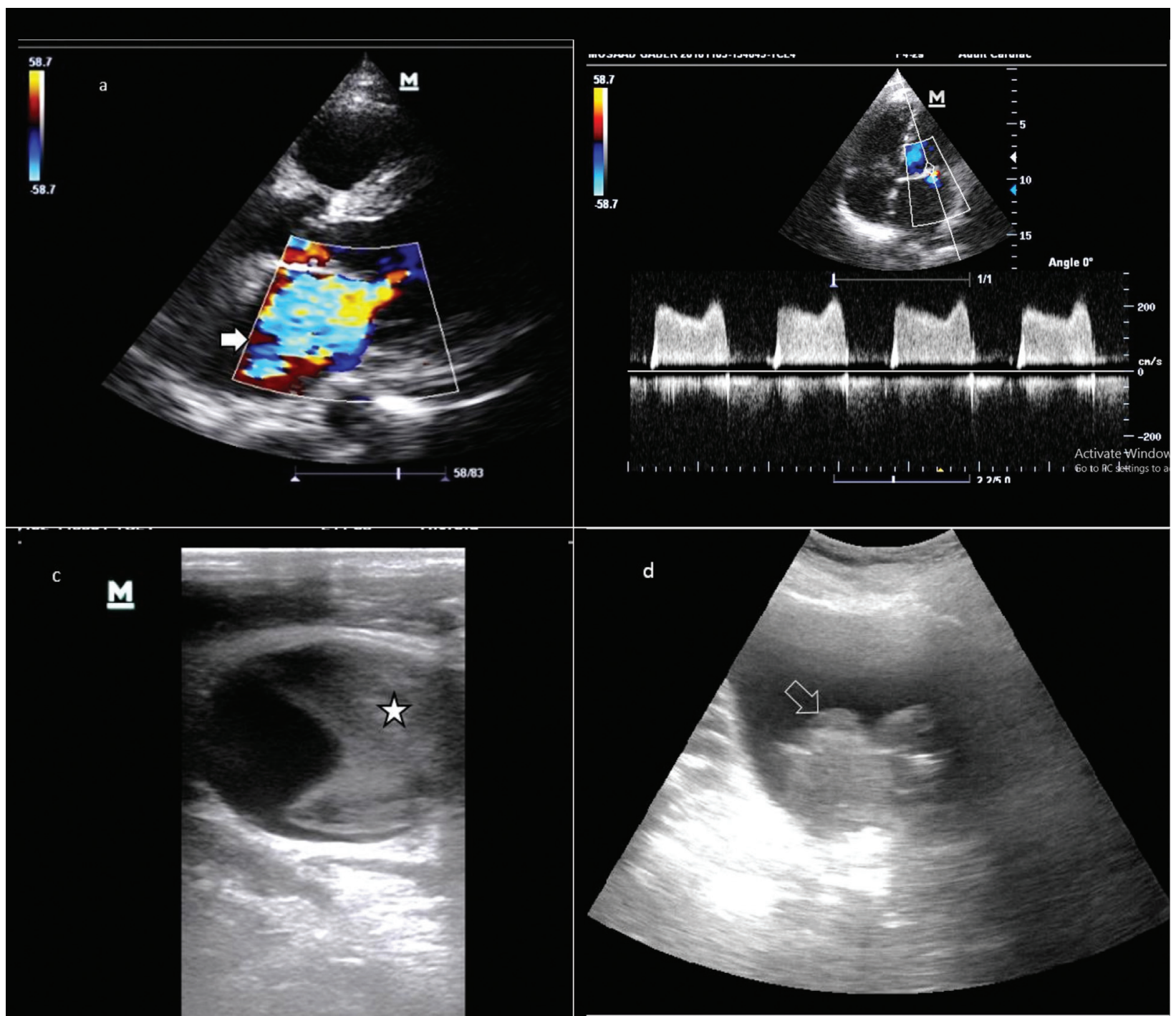

(a) Color Doppler showing severe mitral regurge (arrow) in patient having rheumatic heart disease with pulmonary venous congestion. (b) Continuous-wave Doppler showing severe mitral stenosis [mean pressure gradient (MPG) $17 \mathrm{mmHg}$ and mitral valve area (MVA) $0.8 \mathrm{~cm}^{2}$ by pulmonary hypertension (PHT)] in a patient with systemic lupus presented with hemoptysis. (c) Internal jugular vein thrombosis (star) in a male patient with lung cancer presented with shock. (d) In a 68-year-old male patient with chronic obstructive pulmonary disease presented with acute exacerbation and dysuria, pelviabdominal ultrasound showed a bladder mass (open arrow), which proved to be bladder carcinoma.

ultrasonographic findings prompted further testing in $18.4 \%$ of patients, led to changes in medical therapy in $17.6 \%$ of patients, and led to invasive procedures in $21.6 \%$ patients [2].

\section{Echocardiography}

Transthoracic echocardiography in critically ill has been associated with an improvement in the diagnosis of patients with acute respiratory failure and/or shock [20,21]. It also increased treating physicians ability to pick up subtle acute decompensated heart failure cases initially misdiagnosed as COPD or other diagnoses [8]. Similarly, in our study, echocardiography had the greatest effect on diagnosis in 41\% (42/102) of patients, of whom 13 patients had ejection fraction less than or equal to $45 \%$.

Tricuspid gradient measurement for estimation of right ventricular systolic pressure is a useful and practical method for noninvasive prediction of pulmonary artery pressure and correlates strongly with invasive pulmonary artery systolic pressure assessment [22,23]. A clinical diagnosis of pulmonary hypertension (PHTN) was made if the peak systolic pressure was greater than $30 \mathrm{mmHg}$.

Secondary PHTN is a common complication of chronic lung disease, which is always associated with poor prognosis and usually progresses to right heart 
Figure 2

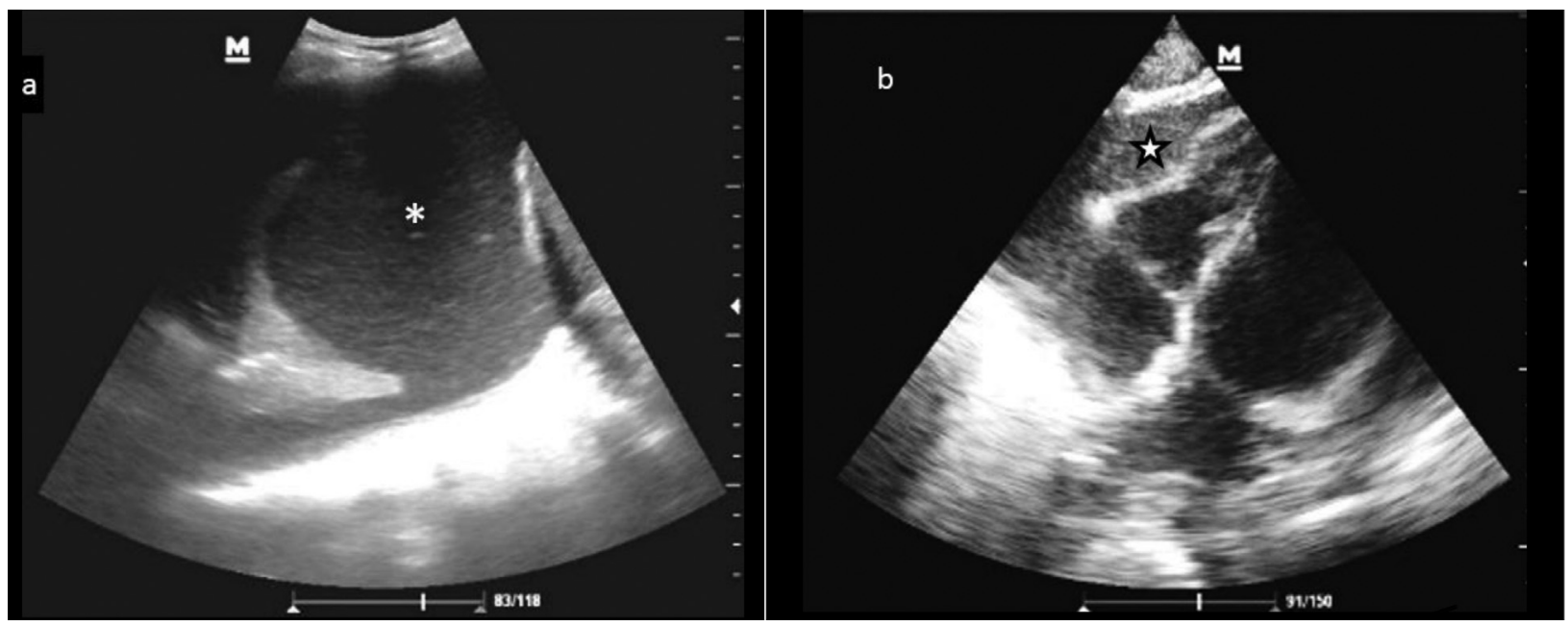

A known breast cancer female patient with severe dyspnea, and chest radiograph showed massive effusion confirmed by lung ultrasound (a). Echocardiography (b) showed pericardial effusion (star) with signs of tamponade adding a new etiological diagnosis for her dyspnea.

Figure 3
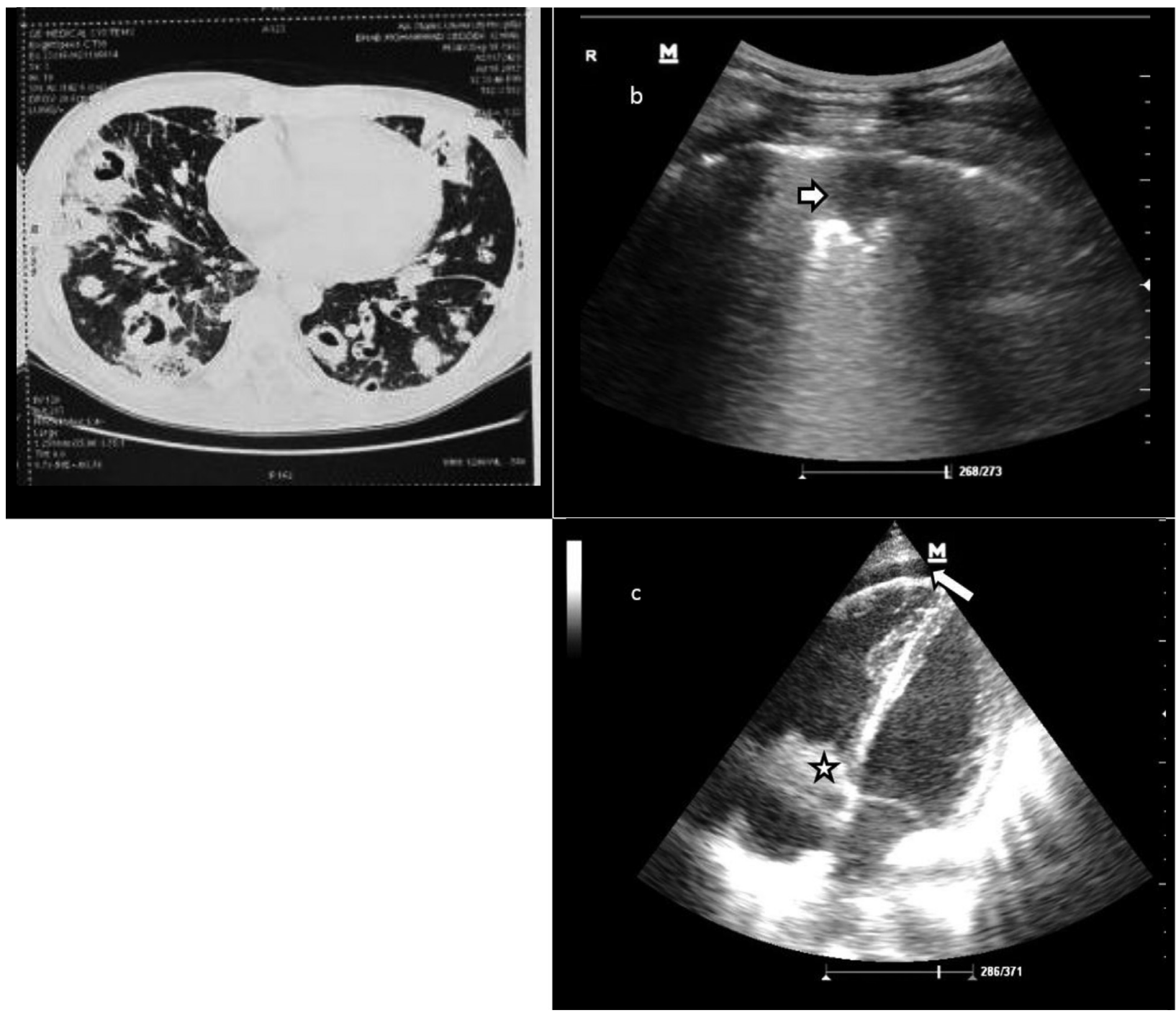

A 35-year-old IV addict male patient presented with toxic symptoms and respiratory failure, and chest radiograph and computed tomography showed septic emboli (a). Lung ultrasound (b) revealed confirmed pleural-based hypoechoic lesions (arrow); echo showed vegetation over tricuspid valve (star) and rim of pericardial effusion (arrow), leading to modification of diagnosis and medical treatment. 
Table 6 The impact of ultrasound examination among studied patients

\begin{tabular}{|c|c|}
\hline & $\begin{array}{c}N=102[n \\
(\%)]\end{array}$ \\
\hline Ultrasound modification of admitting diagnosis & $52(51)$ \\
\hline Echocardiography & $42(41.2)$ \\
\hline Pulmonary hypertension +/- RV dysfunction & $19(18.6)$ \\
\hline Systolic heart failure & $13(12.7)$ \\
\hline Atrial invasion by mediastinal mass & $1(1.0)$ \\
\hline Severe mitral stenosis & $1(1.0)$ \\
\hline Rheumatic heart & $1(1.0)$ \\
\hline Severe mitral regurgitation & $1(1.0)$ \\
\hline Valve vegetations & $2(2.0)$ \\
\hline Moderate pericardial effusion & $2(2.0)$ \\
\hline Tamponading pericardial effusion & $2(2.0)$ \\
\hline Lung ultrasound & $12(11.7)$ \\
\hline Undetected complex effusion & $1(1.0)$ \\
\hline Complex septated effusion & $2(2.0)$ \\
\hline Cardiogenic pulmonary edema pattern & $6(5.9)$ \\
\hline Interstitial lung disease pattern & $2(2.0)$ \\
\hline Focal bronchiectasis & $1(1.0)$ \\
\hline Pelviabdominal ultrasound & $7(6.9)$ \\
\hline Bilateral hydronephrosis & $1(1.0)$ \\
\hline Bladder mass & $1(1.0)$ \\
\hline Hepatic focal lesion(s) & $2(2.0)$ \\
\hline Tense ascites & $2(2.0)$ \\
\hline Chronic calculous cholecystitis & $1(1.0)$ \\
\hline Duplex & $5(4.9)$ \\
\hline Lower limb DVT & $3(2.9)$ \\
\hline IJV thrombosis & $2(2.0)$ \\
\hline More than one examination & $14(13.7)$ \\
\hline Ultrasound confirmed primary diagnosis & $36(35.3)$ \\
\hline Ultrasound wrong diagnosis & $2(2.0)$ \\
\hline Full stomach as abdominal collection & $1(1.0)$ \\
\hline Suspected PE & $1(1.0)$ \\
\hline Ultrasound missed a diagnosis & $12(11.7)$ \\
\hline Missed hilar LN & $2(2.0)$ \\
\hline Missed hilar MASS & $2(2.0)$ \\
\hline Missed mediastinal mass & $1(1.0)$ \\
\hline Missed endobronchial mass & $1(1.0)$ \\
\hline Early ILD & $1(1.0)$ \\
\hline Early apical pneumonia-hemoptysis & $1(1.0)$ \\
\hline Missed small hepatic focal lesions & $1(1.0)$ \\
\hline Missed contralateral pulmonary embolism & $1(1.0)$ \\
\hline Fungal ball & $1(1.0)$ \\
\hline Miliary nodules & $1(1.0)$ \\
\hline $\begin{array}{l}\text { Ultrasound not effective in confirming or } \\
\text { modifying }\end{array}$ & $30(29.4)$ \\
\hline Lead to changes in medical therapy & $42(41.2)$ \\
\hline Intravenous fluids & $10(9.8)$ \\
\hline Diuretics & $12(11.7)$ \\
\hline Diuretics+inotropes & $1(1.0)$ \\
\hline Inotropes & $1(1.0)$ \\
\hline Antiheart failure medications & $5(4.9)$ \\
\hline Anticardiac ischemic & $5(4.9)$ \\
\hline Thrombolytic therapy & $2(2.0)$ \\
\hline Anticoagulation & $4(3.9)$ \\
\hline Antibiotics for infective endocarditis & $1(2.0)$ \\
\hline Promote further investigation & $\begin{array}{l}11(10.8) \\
\text { (Continued) }\end{array}$ \\
\hline
\end{tabular}

Table 6 (Continued)

\begin{tabular}{lc}
\hline & $\begin{array}{c}N=102[n \\
(\%)]\end{array}$ \\
\hline $\begin{array}{l}\text { CT pulmonary angiography } \\
\text { Triphasic computed tomography abdomen }\end{array}$ & $2(2.0)$ \\
scan & $3(2.9)$ \\
Coronary angiography & $2(2.0)$ \\
Transesophageal echocardiography & $2(2.0)$ \\
High resolution CT chest & $1(2.0)$ \\
Further invasive procedure & $14(13.7)$ \\
Percutaneous coronary intervention & $2(2.0)$ \\
Pleural biopsy/thoracoscopy & $1(1.0)$ \\
Fiberoptic bronchoscopy & $1(1.0)$ \\
Ascites tapping & $2(2.0)$ \\
Thoracentesis & $2(2.0)$ \\
Pericardiocentesis & $2(2.0)$ \\
Transthoracic biopsy & $1(1.0)$ \\
Fine needle aspiration cytology & $1(1.0)$ \\
Intercostal tube insertion/VATS & $2(2.0)$
\end{tabular}

DVT, deep venous thrombosis; IJV, internal jugular vein; PHTN, pulmonary hypertension.

failure [24]. However, diagnosis may be delayed in many cases as the dyspnea is usually attributed to the primary lung disease [24]. This was clearly obvious in our study, as 14 (74\%) of 19 patients were newly diagnosed as having PHTN and/or right-sided heart dysfunction, and PHTN was secondary to their primary lung disease (nine patients with COPD, four patients with bronchiectasis, one patient with interstitial lung disease). This finding greatly influenced the workup of the patients and had a direct effect on the therapeutic plan.

Noncardiologists who learn focused critical care echocardiography can adequately interpret basic information and successfully incorporate it into advanced cardiopulmonary life support [25]. On the contrary, noncardiologists with minimal training failed to identify important cardiac abnormalities such as valvular heart diseases, regional wall abnormalities, and acute cor pulmonale [26,27]. This obstacle was surmounted in our study by the comprehensive training given to our sonographer, where severe mitral stenosis as a cause of massive hemoptysis was diagnosed in a patient with known systemic lupus erythematosus and severe mitral regurgitation causing pulmonary venous congestion in another patient, in addition to the previously stated patients with PHTN (Fig. 1).

A variety of complications attributed to septic pulmonary emboli have been described in right-sided endocarditis [28]. This makes transthoracic echocardiography an important initial investigation 
in these patients. POCUS enabled rapid, bedside, noninvasive diagnosis of infective endocarditis in two patients with history of intravenous drug abuse who presented with septic embolic and empyema (Fig. 3).

These data highlight the importance of transthoracic point-of-care echocardiography by respiratory intensivists, as performing an echocardiography and getting immediate interpretation by a cardiologist is not always available in the RICU [29].

\section{Inferior vena cava ultrasound}

Integrating the IVC analysis with a multiorgan ultrasound approach, which includes evaluation of the dimensions and function of the right and left cardiac chambers, with basic evaluation of the pulmonary congestion by assessing lung ultrasound for $\mathrm{B}$ lines led to changes in the medical therapy in $10 / 102(8 \%)$ of our patients. This is consistent with the results of other studies that reported fluid status adjustments to be a frequent therapeutic action performed following a focused ultrasound examination [12].

\section{Lung ultrasound}

In one study, focused LUS identified a missed life-threatening condition in 23 (17\%) patients presenting with acute respiratory symptoms [3]. In another study, LUS pointed out 55 (40.3\%) new findings in patients admitted to medical ICU enabling the differentiation of the etiologic diagnosis in patients with an admitting diagnosis of acute respiratory insufficiency [2].

In our study, LUS was able to assess the lungs in all included patients. A total of 130 findings were seen, of which 12 were identified as new findings; causing modification of the admitting diagnosis in 12/102 (11.7\%) patients. LUS also led to changes in medical therapy in $23(22.5 \%)$ patients, including fluid management and diuretic therapy. We attributed this small number of new finding to the nature of our patients, as most of them have a significant respiratory disorder that was usually apparent in plain chest radiograph.

We describe a pattern of bronchiectasis in LUS, in the form of intersecting comet tail artifacts not erasing A-lines with or without an irregular pleural line detected by linear probe, a finding that was not previously mentioned and needs further analysis (VEDIO).
Figure 4

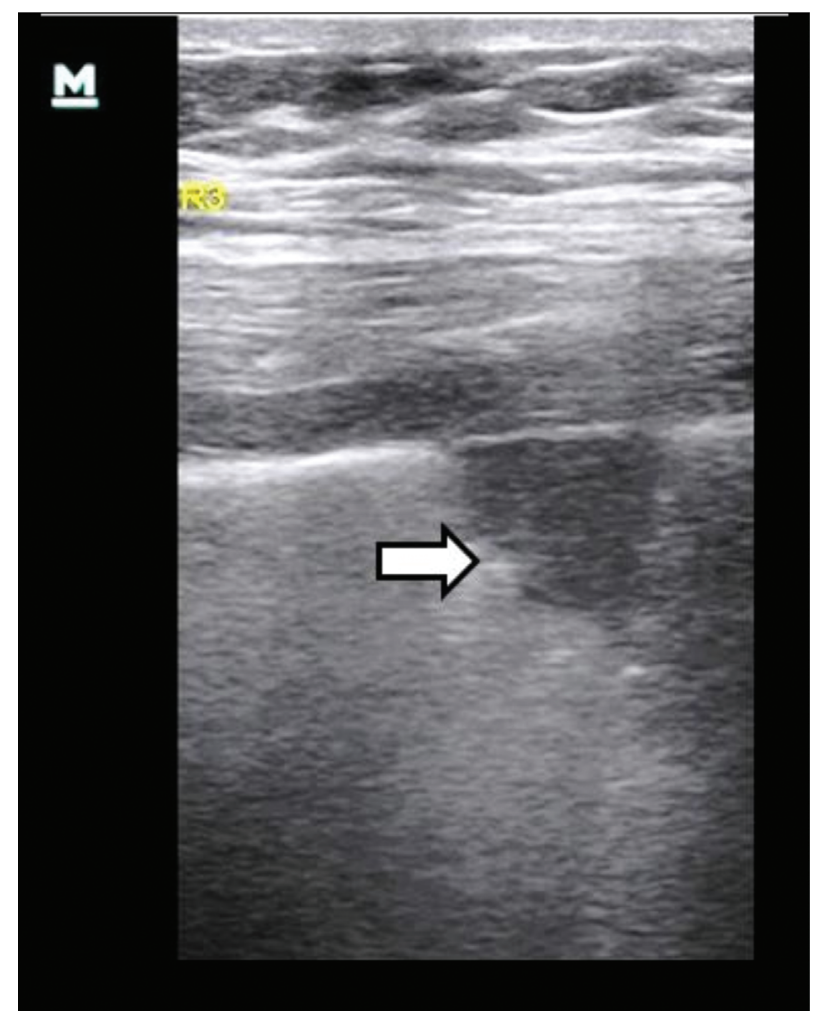

A peripheral hypoechoic lesion (arrow), which was suspected to be a pulmonary infarction in a patient with chronic obstructive pulmonary disease, computed tomography pulmonary angiogram showed an atelectatic band.

At bedside, initial chest ultrasound is more sensitive than chest radiographies in the detection of small pleural effusions that are misdiagnosed as parenchymal opacities or are not seen [9,30]. A total of 32 pleural effusions were detected in our study (Fig. 2), and with the help of bedside LUS, the nature of the fluid could be assessed and aided the change in the management in three cases. Overall, LUS had a greater effect on the therapeutic plan of our patients rather than a diagnostic effect.

\section{Pelviabdominal ultrasound}

Abdominal ultrasound evidenced 14 new pathologic findings and modified admitting diagnosis in 7/102 (6.8\%) cases (Fig. 1). This nearly matched the results of Manno et al. [2], where abdominal examination as a part of the ICU 'sound protocol' evidenced 20 new pathologic findings and induced changes in therapy in $3 / 125(2.4 \%)$ cases.

\section{Duplex}

Central line insertion is a daily practice in ICU. Diagnosing IJV thrombosis in two patients using ultrasonography helped in selecting the site of 
Figure 5
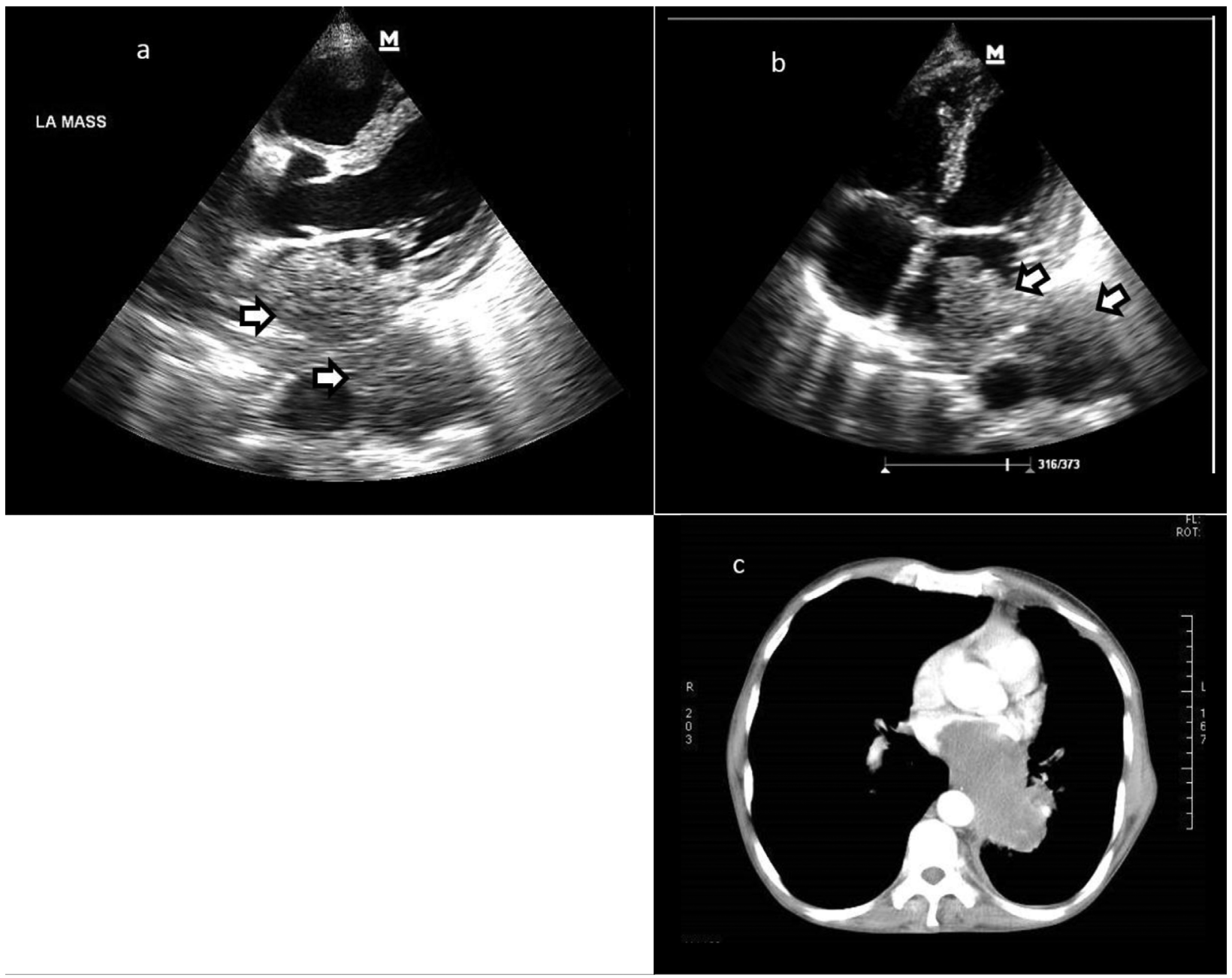

$(a, b)$ Echocardiography showed a mediastinal mass (arrows) with atrial invasion in a patient presented with severe dyspnea, hemoptysis, and wide mediastinum. Computed tomography with contrast (c) was done to identify boundaries of the lesion.

\section{Figure 6}

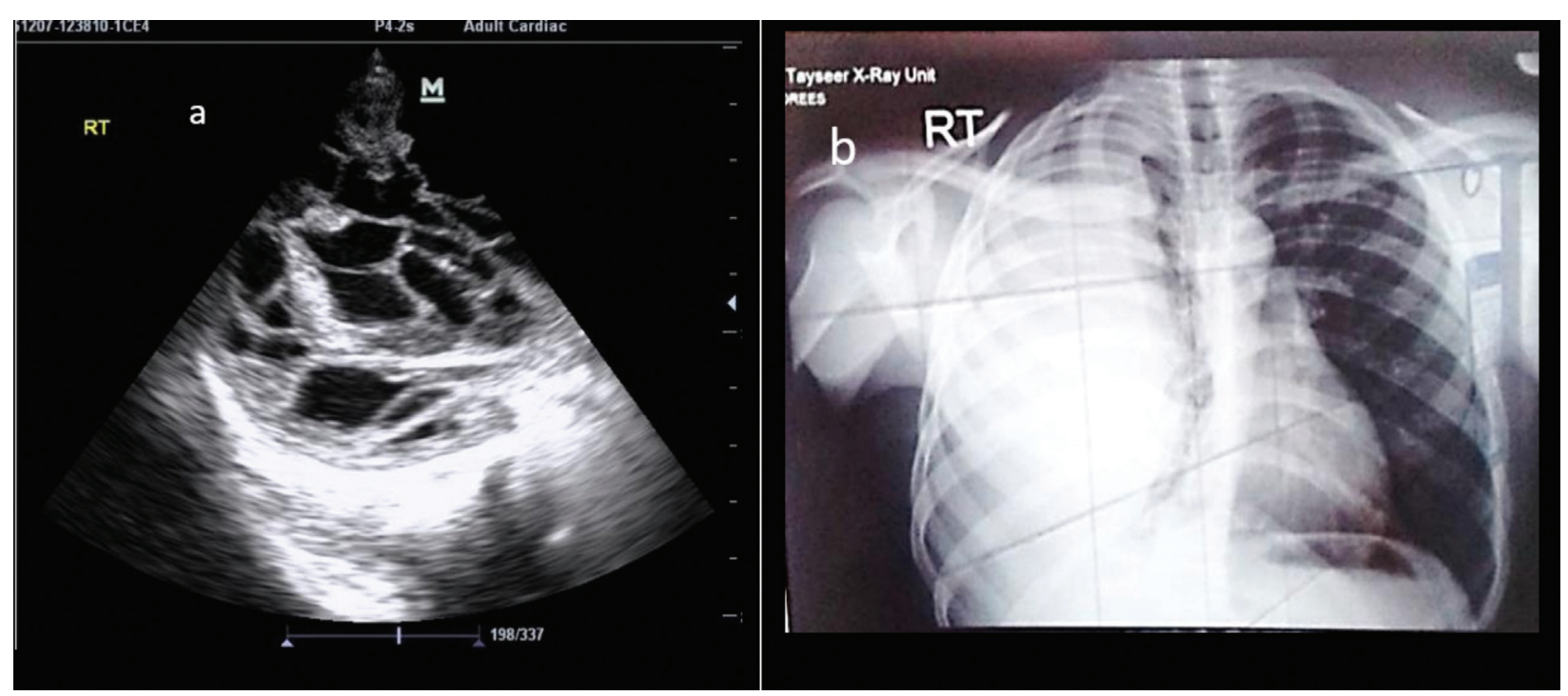

A 30-year-old male presented with severe dyspnea, fever, and right sided D-shaped homogenous opacity (b). Lung ultrasound revealed complex septated pleural effusion (a) managed by medical treatment followed by video-assisted thoracoscopy. 
central line insertion in addition to initiation of therapeutic anticoagulation (Fig. 1).

Among the three patients in which a new lower limb DVT was diagnosed, two cases were diagnosed as having pulmonary embolism. This is in accordance with previous studies that combined echocardiography and venous ultrasonography to chest sonography as a reliable method for screening patients with suspected pulmonary embolism at bedside [31].

\section{Wrong diagnosis}

In two cases, ultrasonography provided a wrong diagnosis; a full stomach appearing below the diaphragm was misdiagnosed as a peritoneal fluid collection, which is a common pitfall in point-ofcare ultrasound [32]. Another patient with systolic heart failure, a pleural-based triangular wedgeshaped opacity suggested the diagnosis of pulmonary embolism (Fig. 4), but appeared to be an atelectatic band in computed tomography pulmonary angiography.

\section{Missed diagnosis}

Transthoracic ultrasound achieves only poor visualization of the mediastinum as compared with computed tomography scan [33]. Thus, it was expected to miss central, mediastinal, or hilar lesions in $5 / 102(4.9 \%)$ of studied patients.

The limitation of our study was that the examiner has not been blinded to the clinical picture of the patient, which is difficult to eliminate in any ultrasound examination. Being a single-center study, which included only patients with respiratory diseases, the results cannot necessarily be applied to other ICUs.

In conclusion, integrating POCUS in the initial assessment of critically ill RICU patients together with standard diagnostic tests led to diagnostic and therapeutic changes in most of patients, which affected these patients' management. Thus, it seems reasonable to consider routine use of POCUS as a new respiratory examination option in the armamentarium of the intensivists.

\section{Financial support and sponsorship Nil.}

\section{Conflicts of interest}

There are no conflicts of interest.

\section{References}

1 Leuppi JD, Dieterle T, Koch G, Martina B, Tamm M, Perruchoud AP, et al. Diagnostic value of lung auscultation in an emergency room setting. Swiss Med Wkly 2005; 135:520-524.

2 Manno E, Navarra M, Faccio L, Motevallian M, Bertolaccini L, Mfochivè A et al. Deep impact of ultrasound in the intensive care unit: the 'iCU-sound' protocol. Anesthesiology 2012; 117:801-809.

3 Laursen CB, Sloth E, Lambrechtsen J, Lassen AT, Madsen PH, Henriksen $\mathrm{DP}$, et al. Focused sonography of the heart, lungs, and deep veins identifies missed life-threatening conditions in admitted patients with acute respiratory symptoms. Chest 2013; 144:1868-1875.

4 Lighthall GK, Vazquez-Guillamet C. Understanding decision making in critical care. Clin Med Res 2015; 13:156-168.

5 Laursen CB, Sloth E, Lassen AT, Christensen R, Lambrechtsen J, Madsen $\mathrm{PH}$, et al. Point-of-care ultrasonography in patients admitted with respiratory symptoms: a single-blind, randomised controlled trial. Lancet Respir Med 2014; 2:638-646.

6 Bhagra A, Tierney DM, Sekiguchi H, Soni NJ. Point-of-care ultrasonography for primary care physicians and general internists. Mayo Clin Proc 2016; 91:1811-1827.

7 Narasimhan M, Koenig SJ, Mayo PH. A whole-body approach to point of care ultrasound. Chest 2016; 150:772-776.

8 Mantuani D, Frazee B, Fahimi J, Nagdev A. Point-of-care multi-organ ultrasound improves diagnostic accuracy in adults presenting to the emergency department with acute dyspnea. West J Emerg Med 2016 17:46-53.

9 Khalil MM, ELMaraghy AA, Yousef YR. Could chest ultrasonography replace routine chest $\mathrm{X}$-rays in mechanically ventilated patients? Egypt $J$ Chest Dis Tuberc 2015; 64:857-863.

10 Salah H. Diagnostic impact of integrating ultrasonography into routine practice in respiratory intensive care units. Egypt $J$ Bronchol 2014; 8:66

11 Bobbia X, Hansel N, Muller L, Claret PG, Moreau A, Genre Grandpierre R, et al. Availability and practice of bedside ultrasonography in emergency rooms and prehospital setting: a French survey. Ann Fr Anesth Reanim 2014; 33:e29-e33.

12 Bernier-Jean A, Albert M, Shiloh AL, Eisen LA, Williamson D, Beaulieu Y. The diagnostic and therapeutic impact of point-of-care ultrasonography in the intensive care unit. J Intensive Care Med 2017; 32:197-203.

13 Zieleskiewicz L, Muller L, Lakhal K, Meresse Z, Arbelot C, Bertrand P-M , et al... Point-of-care ultrasound in intensive care units: assessment of 1073 procedures in a multicentric, prospective, observational study. Intensive Care Med 2015; 41:1638-1647.

14 Gall JR, Lemeshow S, Saulnier F. A New Simplified Acute Physiology Score (SAPS II) based on a European/North American multicenter study. $J$ Am Med Assoc 1993; 270:2957-2963.

15 Lichtenstein DA. BLUE-protocol and FALLS-protocol: two applications of lung ultrasound in the critically ill. Chest 2015; 147:1659-1670.

16 Bahner D, Blaivas M, Cohen HL, Fox JC, Hoffenberg S, Kendall J, et al. AIUM practice guideline for the performance of the focused assessment with sonography for trauma (FAST) examination. J Ultrasound Med 2014 33:2047-2056

17 Ilyas A, Ishtiaq W, Assad S, Ghazanfar H, Mansoor S, Haris M, et al. Correlation of IVC diameter and collapsibility index with central venous pressure in the assessment of intravascular volume in critically ill patients. Cureus 2017; 9:e1025.

18 Volpicelli G, Lamorte A, Tullio M, Cardinale L, Giraudo M, Stefanone V, et al. Point-of-care multiorgan ultrasonography for the evaluation of undifferentiated hypotension in the emergency department. Intensive Care Med 2013; 39:1290-1298.

19 Ghoneim AHA, Hussein RM, El-Ghamry R, Mahmoud LY. Patterns of admitted cases to Respiratory Intensive Care Unit at Zagazig University Hospitals, Egypt. Egypt J Chest Dis Tuberc 2013; 62:661-668.

20 Joseph MX, Disney PJS, Da Costa R, Hutchison SJ. Transthoracic echocardiography to identify or exclude cardiac cause of shock. Chest 2004; 126:1592-1597.

21 Bataille B, Riu B, Ferre F, Moussot PE, Mari A, Brunel E, et al. Integrated use of bedside lung ultrasound and echocardiography in acute respiratory failure: a prospective observational study in ICU. Chest 2014; 146:1586-1593.

22 Forfia $\mathrm{P}$, Roberts J. Diagnosis and assessment of pulmonary vascular disease by Doppler echocardiography. Pulm Circ 2011; 1:160.

23 Chan KL, Currie PJ, Seward JB, Hagler DJ, Mair DD, Jamil Tajik A. Comparison of three Doppler ultrasound methods in the prediction of pulmonary artery pressure. J Am Coll Cardiol 1987; 9:549-554. 
24 Zangiabadi A, De Pasquale CG, Sajkov D. Pulmonary hypertension and right heart dysfunction in chronic lung disease. Biomed Res Int 2014; 2014:739674.

25 Oren-Grinberg A, Talmor D, Brown SM. Focused critical care echocardiography. Crit Care Med 2013; 41:2618-2626.

26 Melamed R, Sprenkle MD, Ulstad VK, Herzog CA, Leatherman JW. Assessment of left ventricular function by intensivists using hand-held echocardiography. Chest 2009; 135:1416-1420.

27 Labbé V, Ederhy S, Pasquet B, Miguel-Montanes R, Rafat C, Hajage D, et al. Can we improve transthoracic echocardiography training in noncardiologist residents? Experience of two training programs in the intensive care unit. Ann Intensive Care 2016; 6:44.

28 Moss R. Injection drug use and right sided endocarditis. Heart 2003; 89:577-581
29 Casaroto E, Mohovic T, Pinto LM, de Lara TR. Bedside echocardiography in critically ill patients. Einstein (São Paulo) 2015; 13:644-646.

30 Kitazono MT, Lau CT, Parada AN, Renjen P, Miller WT. Differentiation of pleural effusions from parenchymal opacities: accuracy of bedside chest radiography. Am J Roentgenol 2010; 194:407-412.

31 Mathis G, Blank W, Reißig A, Lechleitner P, Reuß J, Schuler A, et al. Thoracic ultrasound for diagnosing pulmonary embolism: a prospective multicenter study of 352 patients. Chest 2005; 128:1531-1538.

32 Blanco P, Volpicelli G. Common pitfalls in point-of-care ultrasound: a practical guide for emergency and critical care physicians. Crit Ultrasound J 2016; 8:15.

33 Herth FJF, Becker HD. Transthoracic ultrasound. Respiration 2003; 70:87-94. 